\title{
Discretized knot motion on a tensioned fiber induced by transverse waves
}

\author{
Raffaello Potestio \\ Max Planck Institute for Polymer Research, Ackermannweg 10, 55128 Mainz, Germany* \\ Luca Tubiana \\ Department of Theoretical Physics, Jožef Stefan Institute, \\ Jamova cesta 39, SI-1000 Ljubljana (Slovenia) and \\ University of Vienna, Faculty of Physics, Computational Physics, Sensengasse 8/9 - 1090 Wien, Austri $\oint^{\dagger}$
}

(Dated: Friday $23^{\text {rd }}$ October, 2015)

\begin{abstract}
Topological entanglement is an ubiquitous feature of many biological as well as artificial polymers and fibers. While the equilibrium properties of entangled chains have been the subject of several studies, little is known about their out-of-equilibrium behavior. Here, we address the problem of a stretched knotted fiber driven by a periodic force applied to one of its termini. We show that the onset of standing waves kinetically traps the knot in spatially localized states where the amplitude of the oscillations is maximal, and its normal diffusive dynamics is replaced by discrete jumps.
\end{abstract}

The interest for knots in the biophysics and soft matter community has grown significantly since the seminal works of Frisch and Wasserman [1] and Delbruck [2, who correctly conjectured that knots are ubiquitous in long enough polymers 3 . Since then, knots have been discovered in DNA [4, 5] and proteins [6-10] and have been shown to affect both functional [11 and mechanical [1214 properties of biopolymers. Furthermore, the recently demonstrated possibility of producing knotted solitons in fluid flows [15] and electromagnetic fields [16] as well as that of producing and controlling entangled and knotted disclination lines in liquid crystals with and without colloidal suspensions [17 21] have opened new and exciting prospects for material science.

Novel experimental techniques, e.g. microfluidics apparatuses and last-generation optical tweezer devices $30-$ 34, have now made it possible to directly monitor and investigate the dynamics of macromolecular systems and knotted chains at unprecedented time resolution [12, 3537. Recently, also numerical studies have focused on the dynamics of knots on tensioned polymers in presence of external time-dependent electric fields [29] and fluid flows [38, as well as on the motion of knotted polymers through nanopores or in channels [14, 39]. Of remarkable interest is the emerging picture showing that knots can be actively controlled through external "stimuli", opening the way to their employment in the construction of nano-devices and artificial materials with new properties.

Furthermore the advancement in self-assembling and biomimetic materials opens up the possibility to produce supramolecular fibers in the nano- and micro- scale with exciting mechanical and electronic properties, which can host stable knots 22, 23] or even self assemble [24] or fold themselves 25]27 into a desired knot. The study of topological entanglement in polymers and fibers under non-equilibrium conditions, such as in presence of an external driving force, is therefore a particularly promising

\footnotetext{
* potestio@mpi-mainz.mpg.de

$\dagger$ luca.tubiana@univie.ac.at
}

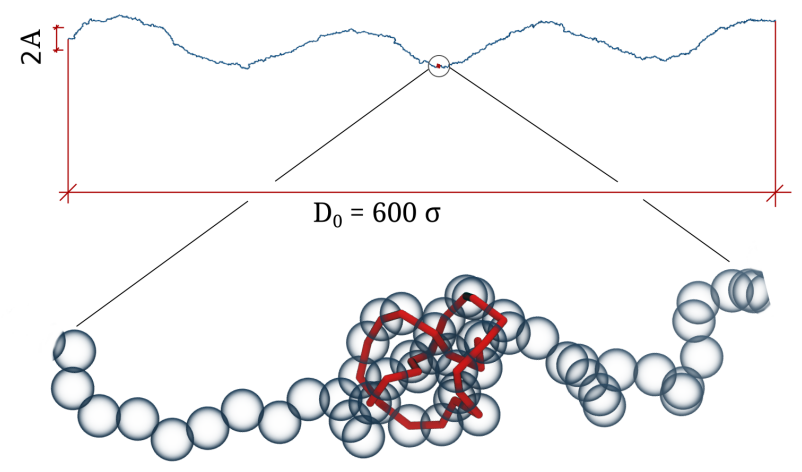

FIG. 1. A snapshot of the tensioned flexible polymer. The $3_{1}$ knot, the simplest possible, is zoomed in in the lower part of the figure.

research field with many possible technological ramifications [14, 28, 29].

Inspired by these possibilities, we studied the response of a knot to the propagation of transverse waves in a stretched fiber. Specifically, we simulated a knotted selfavoiding chain under tension, as illustrated in Fig. 1. In order to consider the phenomenon in its generality we employ a very simplified model, and investigate the behaviour of a fully flexible, self-avoiding chain of $N=1024$ beads stretched along the $X$ axis. Chain connectivity and excluded volume are provided by the FENE [40, 41] bond potential and a purely repulsive Weeks-ChandlerAnderson (WCA) potential [42, respectively. At the chain termini we introduced a "wall" potential, which prevents the knot from untying by passing through one of the ends. This term acts on all beads except for the last 3 near the chain's ends and only in the $X$ direction. The two ends of the chain are restrained to move on two linear guides parallel to $Z$ placed in the origin and in $\left(D_{0}, 0,0\right)$ respectively. The separation $D_{0}$ between the terminal beads was chosen based on the equilibrium properties of knotted chains under tension [12, 35, 43, 46]. Specifically, 


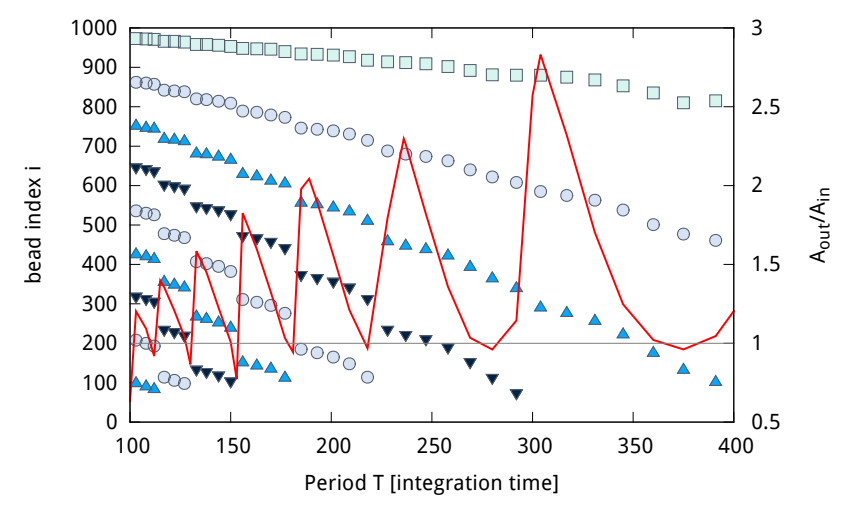

FIG. 2. Quasi-node positions along the chain and transmission coefficient $\mathcal{T}_{f}$, as functions of the forcing period. Data obtained from $\sim 10^{6} \tau_{M D}$ long simulations, performed at 34 different oscillation periods.

we chose a separation $D_{0}=600 \sigma$, with $\sigma$ the diameter of the bead, so that the chain is in the Hookean regime and that the size of the knot remains substantially constant, $\left\langle l_{k}\right\rangle \sim 28 \sigma$, while the knot diffusion time at equilibrium is minimized. The force and diffusion time vs. extension curves are reported as Supporting Information. We drive the first bead sinusoidally, with amplitude $A=10 \sigma$, along the $Z$-axis, generating transverse waves in the $X Z$ plane and leave the last one free to diffuse along $Z$, allowing us to measure easily the transmission of the wave.

The dynamics of the system is described by a Langevin equation with a weak coupling to the solvent $m / \gamma=$ $1000 \tau_{M D}$, where $\tau_{M D}=\sigma \sqrt{m / \epsilon}$ and $\epsilon$ is the strength of the WCA potential. All system setup parameters and results are reported in reduced quantities, where lengths are expressed in units of the bead diameter $\sigma$, the bead mass $m$ and the WCA energy $\epsilon$. The temperature is set to $k_{B} T=\epsilon$. A detailed description of the setup, including the equations for the energy terms implemented and the equation of the dynamics is reported in the supplementary information. The localization of the knot was carried out using the bottom-up knot search strategy and the Minimally Interfering closure described in Tubiana et al. 47.

In order to relate the chain dynamics to the knot motion, we focussed our attention on the following observables of the system: the position $z_{N}$ of the last bead in the $X Z$ plane; the displacements of the beads along $Z$; and the knot motion along the chain.

The chain response to the forced oscillation was monitored by means of the transmission coefficient $\mathcal{T}_{f}$, defined as:

$$
\mathcal{T}_{f}=\frac{\left|\mathcal{F}\left[z_{N}\right]\left(\omega_{f}\right)\right|}{A}
$$

where $\mathcal{F}[\cdot](\omega)$ is the Fourier transform of a timedependent function, $\omega_{f}$ is the frequency of the forced oscillation, and $A$ is the forcing amplitude. In analogy with a forced damped harmonic oscillator [48, we employ
$\mathcal{T}_{f}$ to assess if $\omega_{f}$ is close to a characteristic frequency of the system, i.e. if the chain is resonating with the forcing.

A second measure of the chain response to the driving dynamics is the distribution of the displacement of the beads. Specifically, we consider the root mean square deviation of the $Z$ coordinate of each bead:

$$
\Sigma_{i}=\sqrt{\left\langle\left(z_{i}-\left\langle z_{i}\right\rangle\right)^{2}\right\rangle}
$$

Eq. 2 allows us to identify $B$ chain beads $b_{i}, i \in$ $\{1,2, \cdots B\}$ for which $\Sigma_{i}$ has a local minimum. These beads, termed quasi-nodes, are those which experience the least vertical motion. The term was chosen in analogy with the case of an ideal standing wave: in this case, in fact, the $b_{i}$ 's would identify the wave nodes by construction.

In Fig. 2 we report the bead index of the quasi-nodes and the transmission coefficient for the 34 different values of the forced oscillation period $T_{f}=2 \pi / \omega_{f}$ that we have investigated. Both quantities undergo abrupt jumps for the same values of $T_{f}$. In particular, for increasing period we observe a systematic shift of the quasi-nodes towards the forced bead (having index 1). Also the quasinodes number suddenly changes for specific $T_{f}$ 's exactly in correspondence of a sharp peak in the transmission coefficient.

This behavior is easily rationalized in terms of a systematic onset of standing waves for values of $\omega_{f}$ corresponding to resonating frequencies of the chain. Increasing $T_{f}$ and thus moving away from the resonance, these waves degrade to an irregular motion, until a new resonance is encountered. The change in the number of boundaries is due to the appearance of standing waves of different wavelength, therefore having a different number of nodes. This effect can be seen in the bottom panels of Fig. 3 a) and b), which show the root mean square deviation $\left\langle\Sigma_{i}\right\rangle$ averaged over 12 different simulations for two different periods, one just before the transition, $T_{f}=153 \tau_{M D}$, and one just after, $T_{f}=156 \tau_{M D}$.

In both cases $\left\langle\Sigma_{i}\right\rangle$ shows a steadily growing background due to the diffusion of the free end of the chain. However, while for $T_{f}=153 \tau_{M D}\left\langle\Sigma_{i}\right\rangle$ shows only relatively weak oscillations, for $T_{f}=156 \tau_{M D}$ we observe very pronounced peaks of comparable amplitude. This behavior suggests that in the second case most of the energy given to the system by the forced oscillation is absorbed by only one mode.

The dynamics of the knot can be expected to change when the chain motion ceases to be an irregular fluctuation and turns into a standing wave. This difference is clearly visible in the trajectory of the knot, reported in the top panels of Fig. 3 a) and b). For $T_{f}=153 \tau_{M D}$, Fig. 3 a), the knot diffuses along the chain in a fashion which may appear compatible with a normal diffusion, yet the plot of the probability for a bead to be included in the knot, reported in the bottom panel, shows this dynamics to be distinct from a diffusive one. The probability distribution has a global maximum far from the forced bead, due to the fact that the wave train injected 

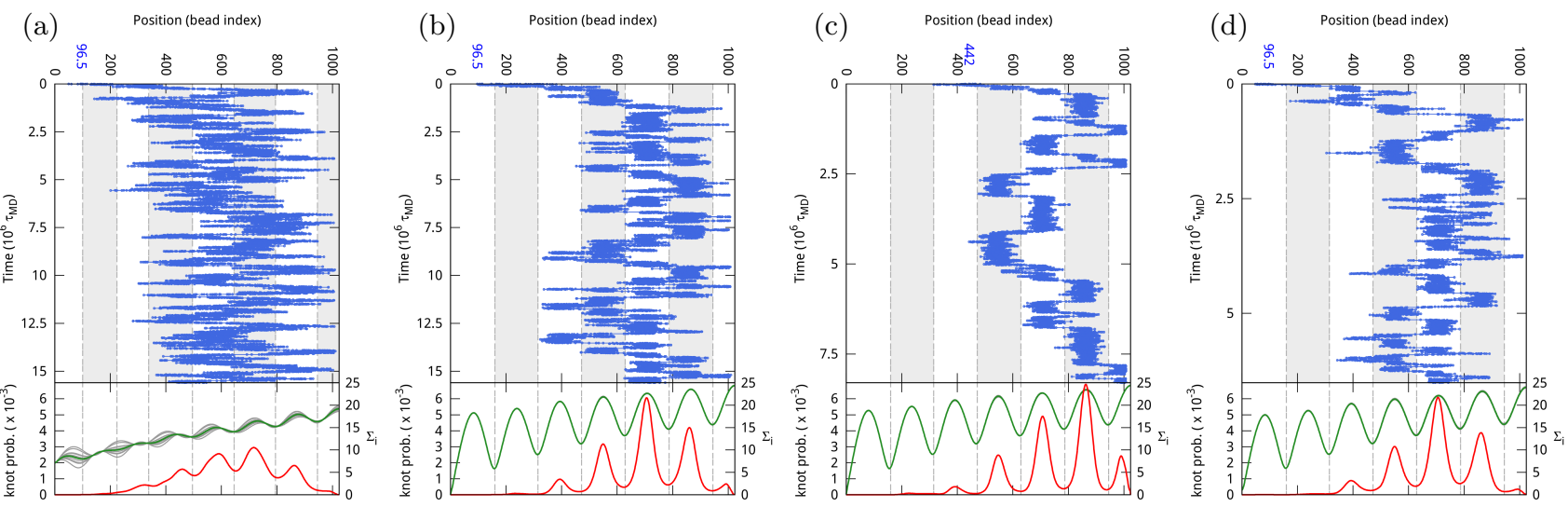

FIG. 3. Knot trajectories and chain dynamics for four different setups. a) $3_{1}$ knot, $T_{f}=153 \tau_{M D}$; b) $3_{1}$ knot, $T_{f}=156 \tau_{M D}$; c) $4_{1}$ knot, $T_{f}=156 \tau_{M D}$; d) $33_{1}$ knot, noisy forcing obtained by adding 4 sub-leading oscillations to the main forcing oscillation at $T_{f}=156 \tau_{M D}$. The top panel of each plot reports the knot center trajectory from one out of several different simulations performed for each case (the initial position of the knots is marked in blue). The bottom panels show: in red, the probability for one bead to be included in the knot, averaged over all trajectories; in green, the root mean square deviation $\left\langle\Sigma_{i}\right\rangle$ averaged over all trajectories; in gray, $\left\langle\Sigma_{i}\right\rangle$, averaged on each trajectory independently. All quantities have been computed over several different trajectories (12, with 6 different knot initial position for panels a) and b); 8 with 4 different knot initial position for panels c) and d)). Movies of part of the trajectories in panels a) and b) are provided as Supporting Information.

in the chain pushes the knot towards the free terminus of the chain, where it is reflected back.

A second and more interesting feature is that the knot position probability distribution shows small but not negligible oscillations, whose maxima coincide with those of the average root mean square deviation $\left\langle\Sigma_{i}\right\rangle$. This feature anticipates a substantially different behavior when the forcing period falls immediately after a resonance.

In fact, for $T_{f}=156 \tau_{M D}$, Fig. $3 \mathrm{~b}$ ), the dynamics of the knot changes dramatically. In this case the knot is almost trapped in specific portions of the chain, delimited by pairs of consecutive quasi-nodes. As shown in the bottom panel of Fig. 3 b), the maxima of $\left\langle\Sigma_{i}\right\rangle$, from now hereafter termed anti-nodes, coincide with the maxima of the knot position distribution. The latter features here much sharper peaks, and is almost zero in correspondence of the quasi-nodes. Consistently, the knot dynamics is characterized by discrete jumps from an anti-node to a neighboring one. The oscillations we have previously observed in the position distribution for $T_{f}=153 \tau_{M D}$ can thus be interpreted as the degraded peaks of the previous resonance. This interpretation is supported by the different number of quasi-nodes measured before and after the transition. In the Supporting Information two videos are provided, showing the different dynamics of both the chain and the knot for the two forcing periods under exam. A completely analogous behavior can be observed for another knot, namely the $4_{1}$, whose discrete dynamics at the resonant frequency is reported in panel c) of Fig. 3 .

The discrete dynamics of the knot upon onset of the standing wave is quite resilient with respect to perturbations of the latter. In panel d) of Fig. 3 we report the trajectory and position distribution of a $3_{1}$ knot subject to a disturbed forcing, where to the resonant oscillations other ones are added, having lower amplitude but close-by frequency. The forcing applied to the system of Fig. 3 d) is in fact the sum of the fundamental forcing $\left(A=10 \sigma, T_{f}=156 \tau_{M D}\right)$ plus four other terms with $A=\sigma, T=156 \pm 1.5 \tau_{M D}$ and $A=0.1 \sigma, T=156 \pm 2 \tau_{M D}$. Also in this case, the confinement between quasi-nodes and the sharp jumps across the latter occur as observed when only a well-defined, sinusoidal forcing is applied.

It is at first counterintuitive that the knot is confined in the anti-nodes, that is, the regions featuring the highest mobility $\left\langle\Sigma_{i}\right\rangle$, rather than remaining stuck in a quasinode, where the chain fluctuates less. The explanation can be found in the dynamical properties of the string in the neighborhood of a standing wave node. The latter, in fact, is by definition a point of the wave whose amplitude is always zero. The spatial derivative of the wave function in those points, however, oscillates more than anywhere else along the chain. The anti-nodes undergo the opposite behavior: their position along $Z$ oscillates the most, but the derivative of the wave vanishes, meaning that all neighboring points move together and, therefore, are almost at rest relatively to each other. As shown in Fig. 3 , the knot is then more favorably located in the anti-node region, as the latter is globally more mobile, but locally more stable.

This line of reasoning can be made more quantitative. Since the length of the knot does not vary sensibly during our simulations, we can model it as a point-like mass, $m$, constrained to move along the chain (see e.g. Di Stefano et al. 29] and Huang and Makarov [44]). We then model the chain as an ideal, sinusoidal standing wave $z$, given by $z(x, t)=A \sin (\omega t) \sin (k x)$, where $k=\left(n+\frac{1}{2}\right) \pi / L$, 
in accord with the fact that our chain has one fixed end and one free end. In fact, it is a well know result 49 that a forced oscillation, imposed at one extreme of a tensioned string having the other end free, induces a sinusoidal standing wave with amplitude exponentially decreasing as a function of the distance from the forced end. Since the decay length is inversely proportional to the friction of the medium, we make use of the weak coupling between our chain and the thermostat to neglect this damping in the model we here discuss.

In absence of an external potential, the Lagrangian of the particle constrained to move on the standing wave coincides with its kinetic energy: $\mathcal{L} \equiv \mathcal{T}=m / 2\left(v_{x}^{2}+v_{z}^{2}\right)$. The Lagrangian equations of motion lead us to:

$$
m \ddot{x}=m v_{z} \frac{\partial v_{z}}{\partial x}=\frac{m k \omega^{2} A^{2}}{2} \cos ^{2}(\omega t) \sin (2 k x)
$$

where we made use of the definition of $z(x, t)$ to compute $v_{z}=\dot{z}$. The time average of the force along $x$ over an oscillation period and the corresponding potential of mean force are:

$$
\begin{aligned}
& \langle F(x)\rangle=\frac{m k \omega^{2} A^{2}}{4} \sin (2 k x) \\
& V(x)=-\int_{0}^{x}\left\langle F\left(x^{\prime}\right)\right\rangle d x^{\prime}=-\frac{m \omega^{2} A^{2}}{4} \sin ^{2}(k x)
\end{aligned}
$$

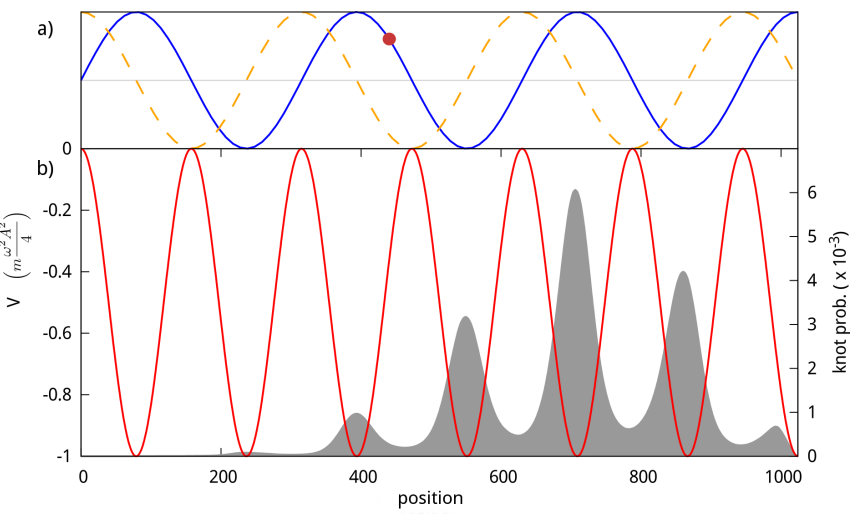

FIG. 4. a) Scheme of the theoretical model used to analyze the behavior of the oscillating knotted chain. The chain is represented as an ideal standing wave (solid blue line) on which a point-like mass representing the knot can move (red disc). The dashed orange line shows the position derivative of the standing wave. b) Comparison between the potential of mean force (Eq. 5 corresponding to a standing wave with $n=6$ nodes and the probability for one bead to be included in the knot obtained for $T_{f}=156 \tau_{M D}$.

In summary, we have shown that the dynamics of a knot on a tensioned fiber becomes discrete when the chain is forced to oscillate at its resonating frequency. Specifically, the knot becomes localized in the anti-nodes, that is, the globally most mobile and locally more stable regions of the chain. The motion between anti-nodes does not occur by a slow diffusive-like motion, rather by means of discrete jumps. Analogous behavior is observed also for the next-to-simplest topological entanglement, the $4_{1}$ knot as well as when the chain is subjected to a noisy forcing oscillation. Furthermore, while in our case we considered for simplicity a chain in which the right end is free to move along the $Z$ axis, we expect the same behaviour to occur in a system setup in which the right end is fixed: in fact, the only impact the different boundary condition would have is on the wavelength of the fundamental oscillation and, hence, of the resonating frequency.

These results demonstrate how the onset of an outof-equilibrium steady state in a knotted chain can be exploited to induce a qualitatively different steady-state dynamics of a knot. Potential applications may span from the localization of topological entanglements on microscopic and macroscopic fibers to their control and use for technological purposes.

\section{ACKNOWLEDGMENTS}

The authors are indebted to C. Micheletti and I. Coluzza for insightful discussions and a careful reading of the manuscript. LT acknowledges support from the Slovenian Agency for Research and Development (ARRS grant No J1-4134) and from the MahlkeOberman Stiftung and the European Union's Seventh Framework Programme for research, technological development and demonstration (grant No 609431).
As shown in Fig. 4 this effective potential has maxima in correspondence of the standing wave nodes, and minima in correspondence of the anti-nodes, coherently with the observed behavior of the knot. 
[1] E. Frisch, H.L and Wasserman, Journal of the American Chemical Society, 1961, 83, 3789-3795.

[2] M. Delbrück, Mathematical problems in biological sciences, 1962, p. 55.

[3] D. Sumners and S. Whittington, Journal of Physics A: Mathematical and General, 1988, 21, 1689.

[4] L. Liu, R. Depew and J. Wang, Journal of molecular biology, 1976, 106, 439-452.

[5] L. F. Liu, L. Perkocha, R. Calendar and J. C. Wang, Proceedings of the National Academy of Sciences of the United States of America, 1981, 78, 5498-5502.

[6] W. Taylor, Nature, 2000, 406, 916-919.

[7] P. Virnau, L. A. Mirny and M. Kardar, PLoS Comput Biol, 2006, 2, e122.

[8] A. L. Mallam, J. M. Rogers and S. E. Jackson, Proceedings of the National Academy of Sciences, 2010, 107, 8189-8194.

[9] R. Potestio, C. Micheletti and H. Orland, PLoS computational biology, 2010, 6, e1000864.

[10] T. Škrbić, C. Micheletti and P. Faccioli, PLoS computational biology, 2012, 8, e1002504.

[11] J. Portugal and A. Rodríguez-Campos, Nucleic acids research, 1996, 24, 4890-4894.

[12] Y. Arai, R. Yasuda, K. Akashi, Y. Harada, H. Miyata, T. Kinosita and H. Itoh, Nature, 1999, 399, 446-448.

[13] A. M. Saitta, P. D. Soper, E. Wasserman and M. L. Klein, Nature, 1999, 399, 46-48.

[14] A. Rosa, M. Di Ventra and C. Micheletti, Physical Review Letters, 2012, 109, 118301.

[15] D. Kleckner and W. T. Irvine, Nature Physics, 2013, 9, 253-258.

[16] H. Kedia, I. Bialynicki-Birula, D. Peralta-Salas and W. T. M. Irvine, Phys. Rev. Lett., 2013, 111, 150404.

[17] U. Tkalec, M. Ravnik, S. Čopar, S. Žumer and I. Muševič, Science, 2011, 333, 62-65.

[18] B. Senyuk, Q. Liu, S. He, R. D. Kamien, R. B. Kusner, T. C. Lubensky and I. I. Smalyukh, Nature, 2013, 493, 200-205.

[19] T. Machon and G. P. Alexander, Proceedings of the $\mathrm{Na}$ tional Academy of Sciences, 2013, 110, 14174-14179.

[20] A. Martinez, M. Ravnik, B. Lucero, R. Visvanathan, S. Žumer and I. I. Smalyukh, Nature materials, 2014, 13, 258-263.

[21] D. Seč, S. Čopar and S. Žumer, Nature communications, $2014, \mathbf{5}$, year.

[22] X. Hu, Z. Xu and C. Gao, Scientific reports, 2012, 2, year.

[23] X. Yan, S. Li, J. B. Pollock, T. R. Cook, J. Chen, Y. Zhang, X. Ji, Y. Yu, F. Huang and P. J. Stang, Proceedings of the National Academy of Sciences, 2013, 110, 15585-15590.
[24] G. Polles, D. Marenduzzo, E. Orlandini and C. Micheletti, Nature communications, 2015, 6, year.

[25] I. Coluzza, P. D. van Oostrum, B. Capone, E. Reimhult and C. Dellago, Soft Matter, 2013, 9, 938-944.

[26] I. Coluzza, P. D. van Oostrum, B. Capone, E. Reimhult and C. Dellago, Physical review letters, 2013, 110, 075501.

[27] S. Najafi and R. Potestio, PLoS ONE, 2015, 10, e0132132.

[28] X. Jiang, L. Tong, G. Vienne, X. Guo, A. Tsao, Q. Yang and D. Yang, Applied Physics Letters, 2006, 88, 223501.

[29] M. Di Stefano, L. Tubiana, M. Di Ventra and C. Micheletti, Soft Matter, 2014, 10, 6491-6498.

[30] L. Olavarrieta, M. L. Martnez-Robles, P. Hernndez, D. B. Krimer and J. B. Schvartzman, Mol Microbiol, 2002, 46, 699-707.

[31] G. A. Arteca, Theoretical Chemistry Accounts: Theory, Computation, \& Modeling, 2007, 118, 549 - 556.

[32] S. Trigueros and J. Roca, BMC Biotechnol, 2007, 7, 94.

[33] A. Leforestier, S. Brasils, M. de Frutos, E. Raspaud, L. Letellier, P. Tavares and F. Livolant, J Mol Biol, 2008, 384, 730-739.

[34] J. Tang, N. Du and P. S. Doyle, Proceedings of the National Academy of Sciences, 2011, 108, 16153-16158.

[35] X. Bao, H. Lee and S. Quake, Physical review letters, 2003, 91, 265506.

[36] J. Tang, N. Du and P. S. Doyle, Proceedings of the National Academy of Sciences, 2011, 108, 16153-16158.

[37] C. B. Renner and P. S. Doyle, Soft matter, 2015.

[38] C. B. Renner and P. S. Doyle, ACS Macro Letters, 2014, 3, 963-967.

[39] C. Micheletti and E. Orlandini, ACS Macro Letters, 2014, 3, 876-880.

[40] G. S. Grest and K. Kremer, Phys. Rev. A, 1986, 33, 3628-3631.

[41] K. Kremer and G. S. Grest, The Journal of Chemical Physics, 1990, 92, year.

[42] J. D. Weeks, D. Chandler and H. C. Andersen, J. Chem. Phys., 1971, 54, $5237-5247$.

[43] A. Vologodskii, Biophys. J., 2006, 90, 1594.

[44] L. Huang and D. E. Makarov, The Journal of Physical Chemistry A, 2007, 111, 10338-10344.

[45] X. Zheng and A. Vologodskii, Phys. Rev. E, 2010, 81, 041806.

[46] R. Matthews, A. Louis and J. Yeomans, EPL (Europhysics Letters), 2010, 89, 20001.

[47] L. Tubiana, E. Orlandini and C. Micheletti, Progress of Theoretical Physics Supplement, 2011, 191, 192-204.

[48] L. Landau and E. Lifshitz, Mechanics, ButterworthHeinemann, Oxford, 1976.

[49] V. Smirnov, A course of higher Mathematics, Pergamon Press, Oxford, 1964, vol. 2. 